\title{
Fuel-Saving Strategies for Dual Spacecraft Interferometry Missions
}

Christopher A. Bailey

Timothy W. McLain

Brigham Young University - Provo, mclain@byu.edu

Randal W. Beard

Brigham Young University - Provo, beard@byu.edu

Follow this and additional works at: https://scholarsarchive.byu.edu/facpub

Part of the Electrical and Computer Engineering Commons, and the Mechanical Engineering

Commons

\section{Original Publication Citation}

Bailey, C., McLain, T., and Beard, R. Fuel-Saving Strategies for Dual Spacecraft Interferometry Missions, Journal of the Astronautical Sciences, vol. 49, no. 3, pp. 469-488, July-September 2001.

\section{BYU ScholarsArchive Citation}

Bailey, Christopher A.; McLain, Timothy W.; and Beard, Randal W., "Fuel-Saving Strategies for Dual Spacecraft Interferometry Missions" (2001). Faculty Publications. 1539.

https://scholarsarchive.byu.edu/facpub/1539

This Peer-Reviewed Article is brought to you for free and open access by BYU ScholarsArchive. It has been accepted for inclusion in Faculty Publications by an authorized administrator of BYU ScholarsArchive. For more information, please contact ellen_amatangelo@byu.edu. 


\section{American Astronautical Society}

VOLUME 49 , NO. 3

JULY-SEPTEMBER 2001

\section{CONTENTS}

Moon Assisted Out of Plane Maneuvers of Earth Spacecraft

$$
\text { C. Circi, F. Graziani, and P. Teofilatto }
$$

Average and Maximum Revisit Time Trade Studies for Satellite Constellations Using a Multiobjective Genetic Algorithm

$$
\begin{array}{r}
\text { Edwin A. Williams, William A. Crossley, } \\
\text { and Thomas J. Lang }
\end{array}
$$

On the Rigid Rotation Conept in $n$-Dimensional Spaces

$$
\text { Daniele Mortari }
$$

Flexible Spacecraft Reorientations Using Gimbaled Momentum Wheels

Kevin A. Ford and Christopher D. Hall

Mode and Logic-Based Switching for the Formation Flying Control of Multiple Spacecraft

$$
\text { Mehran Mesbahi and Fred Y. Hadaegh }
$$

Fuel-Saving Strategies for Dual Spacecraft Interferometry Missions

$$
\text { Christopher A. Bailey, Timothy W. McLain, }
$$
and Randal W. Beard

On the Information Dilution Theorem and Its Application to Attitude Determination 


\title{
Fuel-Saving Strategies for Dual Spacecraft Interferometry Missions
}

\author{
Christopher A. Bailey, ${ }^{1}$ Timothy W. McLain, ${ }^{2}$ and Randal W. Beard ${ }^{3}$
}

\begin{abstract}
Separated spacecraft interferometry missions will require that spacecraft move in a coordinated fashion to ensure minimal and balanced consumption of fuel. This paper develops strategies for determining interferometry mission plans that result in significant fuel savings over standard approaches. Simulation results demonstrate that valuable reductions in fuel consumption can be realized by combining the retargeting and imaging maneuvers required to image multiple stellar sources. Fuel-optimal imaging strategies have been developed for two-spacecraft interferometry missions similar to the proposed StarLight mission using chained local optimization methods. Based on these strategies, sampling-pattern guidelines for space-borne interferometry missions have been developed.
\end{abstract}

\section{Introduction}

Space-based optical interferometry has been identified by NASA as one of the key technologies in furthering the scientific exploration of the universe in the next century. NASA's Origins Program will use the fundamentals of interferometry to form a sophisticated space-based telescope as the primary tool for future space missions to image stars and distant planetary systems with new levels of accuracy. This paper develops fuel-saving schemes for separated spacecraft stellar interferometry missions. For such missions, the objective is to minimize the amount of propellant mass required to perform the mission objectives while ensuring that no spacecraft in the formation is left with insufficient fuel to complete the mission.

Interferometers image stellar targets in a significantly different way than conventional telescopes, which use a single, large primary mirror. An optical interferometer samples parallel wavefronts at two or more locations and combines the light to produce an interference pattern. From these interference patterns or fringes, high angular resolution images of the source can be constructed. Optical interferometry requires a precisely controlled separation between the light collecting apertures.

'NTT/Verio-SME Hosting, Orem, Utah 84097.

${ }^{2}$ Department of Mechanical Engineering, Brigham Young University, Provo, Utah 84602.

${ }^{3}$ Department of Electrical and Computer Engineering, Brigham Young University, Provo, Utah 84602. 
The resolution of the interferometer is proportional to the largest possible baseline separation.

Shao and Colavita [1] give a comprehensive technical review of ground-based interferometry and the advantages of space-based interferometry. Joshi [2] provides an excellent introduction to interferometry and offers a technical discussion on how images are constructed from interferometric-instrument interference patterns. Figure 1 shows an example of the relationship between the source and the observation plane. The spacecraft collect observations in the form of interference fringes from the source at locations given by $\left(\eta_{1}, \nu_{1}\right)$ and $\left(\eta_{2}, \nu_{2}\right)$ in the observation plane. Joshi [2] explains that the van Cittert-Zernike result is a two-dimensional Fourier transform and is the basis for interferometric imaging. An irradiance pattern, $I(x, y)$, that forms the image of the source $S$ can be constructed from this transform by

$$
I(x, y) \triangleq \int_{-\infty}^{\infty} \int_{-\infty}^{\infty} \mu(u, v) e^{i 2 \pi(u x+v y)} d u d v
$$

The function $\mu(u, v)$ is called the complex mutual coherence function. Points in the $(u, v)$ plane are mapped from locations of the spacecraft in the $(\nu, \eta)$ plane by

$$
\begin{gathered}
u \triangleq \frac{\eta_{1}-\eta_{2}}{z \lambda} \\
v \triangleq \frac{\nu_{1}-\nu_{2}}{z \lambda}
\end{gathered}
$$

where $\lambda$ is the wavelength of emitted light and $z$ is the distance to the source. The mutual coherence function is calculated from amplitude and phase measurements made in the observation plane $(\nu, \eta)$ for each point in the $(u, v)$ plane. Due to the sym-

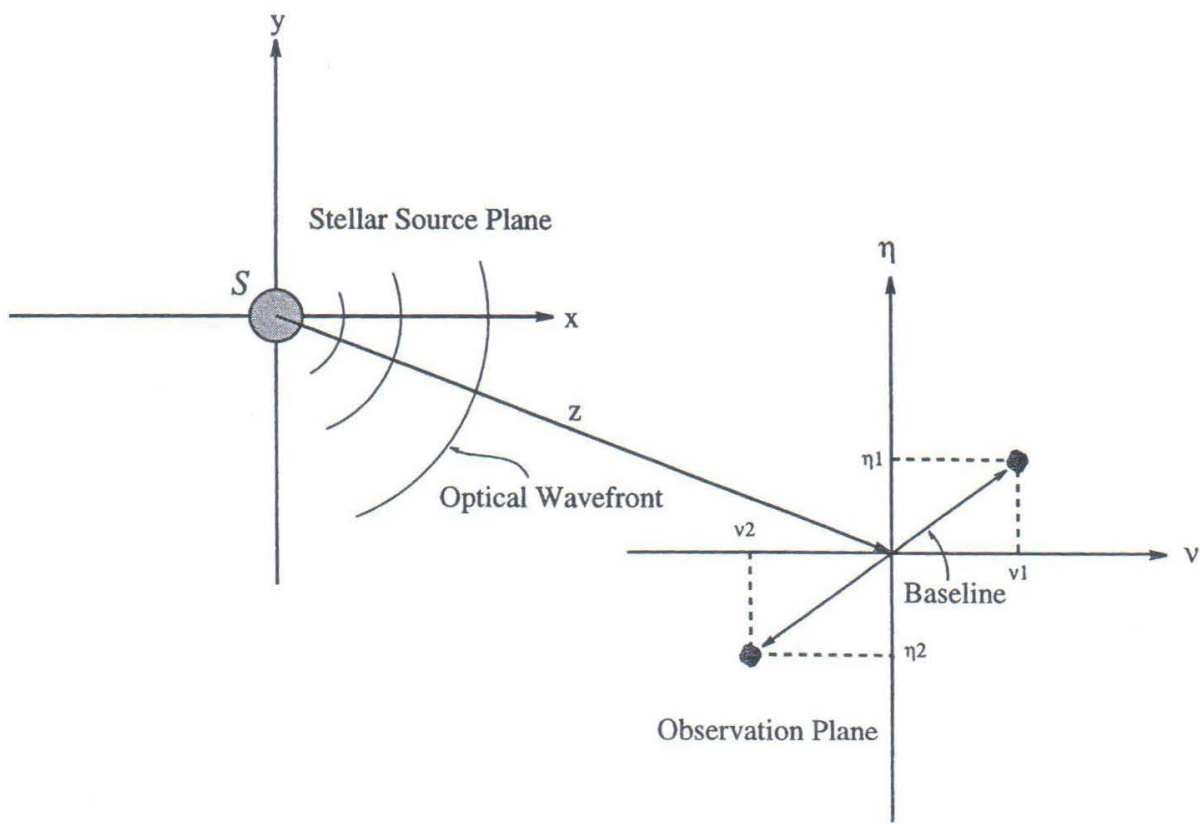

FIG. 1. Synthetic Aperture Imaging Scenario. 
metry properties of Fourier transforms, celestial targets that are spherical (e.g., a single star) require only the sampling of a radial line in the $(u, v)$ plane. These targets are called 1-D targets. Alternatively, nonspherical targets (e.g., a binary star) require sampling of half of the $(u, v)$ plane, and are termed 2-D targets. Throughout this paper, stellar targets are assumed to be referenced in the celestial sphere.

Separated spacecraft formations offer an intriguing platform for space-based interferometry. As Fig. 2 illustrates, interferometry requires three basic formation maneuvers. The first, retargeting, involves realigning the light collecting apertures with a new celestial target. To do so, the spacecraft are rotated about some point in space to redirect the optical bore sight of the interferometer at the new source. The next is reorienting the formation about the bore sight of the interferometer. This provides a degree of freedom used for sampling points in the $(u, v)$ plane at a constant baseline separation between spacecraft. The last is resizing, where the formation maintains the same observation direction while spacecraft move to change the baseline separation. A typical mission will involve a combination of these maneuvers in order to sample the $(u, v)$ plane for a collection of stars.

Interferometry requires that the baseline separation for the spacecraft be maintained to within very tight tolerances (a fraction of the wavelength of light). Laser metrology systems are used to measure the spacecraft separations to this level of accuracy. Initializing the sensor can be a costly procedure in terms of fuel and time. Therefore, it is sometimes desirable to perform spacecraft formation maneuvers that maintain the formation throughout the maneuver. Another strategy is to allow the spacecraft to break formation during the maneuver, thus requiring a laser metrology reinitialization. In this paper, we will assume that the spacecraft can break formation during each maneuver; however, the techniques developed extend easily to the sensor-lock case.

Retarget, reorient, and resize maneuvers all require active thrusting, and therefore they expend onboard propellant, making fuel the major limitation to the length of the mission. It is therefore imperative that formation maneuvers be performed in a way that minimizes the expended fuel. An additional complication, however, is that the useful lifetime of the mission is limited by the first spacecraft to run out of fuel. In performing fuel-minimal retarget maneuvers, one of the spacecraft may burn fuel at a significantly faster rate than the others $[3,4]$. Therefore, an additional goal is to perform formation maneuvers such that the fuel on each spacecraft is equalized.
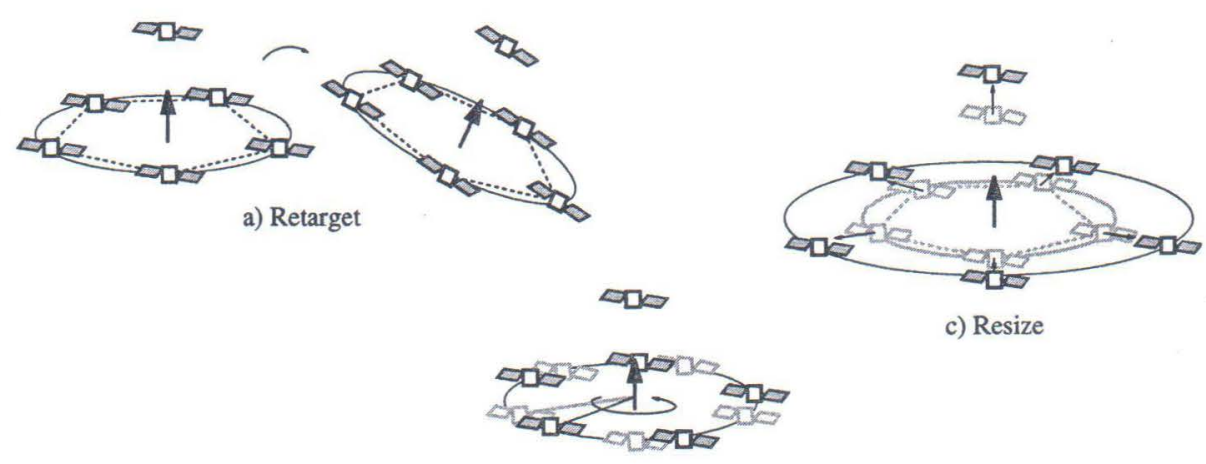

c) Resize

b) Reorient

FIG. 2. Repositioning Maneuvers for Space-Based Optical Interferometry. 
For individual retarget, resize, and reorient maneuvers, it is possible to derive fuel minimizing/equalizing maneuvers. The general fuel minimization/equalization problem has been solved for individual retarget and reorientation maneuvers in [3] for constrained (i.e., sensor-lock) maneuvers, and in [4] for unconstrained maneuvers. In [5], a solution to the fuel-minimization/equalization problem for resize maneuvers in the context of the planned StarLight mission is given.

Equalizing or minimizing the fuel for individual maneuvers may be thought of as a local fuel-optimization problem. In this paper, we are primarily concerned with the global fuel-optimization problem; i.e., given that each individual formation maneuver can be performed in a fuel-minimizing/equalizing manner, how should the maneuvers be sequenced to minimize/equalize the overall fuel usage?

A reasonable approach would be to find the fuel-optimal sequence of retarget maneuvers, assuming that once the formation has been retargeted to a single star, all $(u, v)$ points will be sampled for that star (through a sequence of reorient and resize maneuvers), before retargeting to the next star. However, if the angular separation between two stars is small, then considerable fuel savings can be obtained by mixing $(u, v)$ points from one star with those of another. Therefore, the approach taken here is to solve the optimization problem, allowing arbitrary mixing between reorient, resize, and retarget maneuvers. In other words, from a fuel point of view, it may be better to collect a couple of $(u, v)$ samples from star \#1, retarget, collect a couple $(u, v)$ samples from star \#2, and then retarget back to star \#1 to collect more $(u, v)$ samples for that star. Our results show cases for which this is true.

By allowing retarget, reorient, and resize maneuvers to be mixed, the solution space of the problem is increased considerably. Fortunately, however, our simulation results show some general patterns for how retarget, reorient, and resize maneuvers can be mixed to achieve considerable fuel savings. These patterns can be used to derive simple search heuristics to aid the optimization algorithm or to guide the determination of targeting and imaging sequences for specific imaging missions.

\section{Background}

\section{Space-based Interferometry and Spacecraft Formation Control}

A significant level of research activity on space-based interferometry has been maintained in recent years. A brief survey of the current literature on spacecraft formation control with specific application to space-based interferometry is given here.

An overview of the New Millennium separated spacecraft interferometer Deep Space 3 mission is given in [6]. Budget limitations required changes to be made to the Deep Space 3 mission. The changes reflected in the new concept of the renamed StarLight mission are described in [7].

Various strategies have been explored for formation control. Leader-following strategies have been developed in [8,9]. Behavioral approaches are discussed in [10]. In [11], formation-rotation controls are developed using adaptive control, considering the presence of actuator saturation and small changes in the mass properties of the spacecraft. A new architecture for the coordinated control of spacecraft formations is introduced in [12], which includes leader-following, behavioral, and virtual-structure approaches. A framework for adaptive control of relative spacecraft position is given in [13]. 


\section{StarLight Mission}

While the methods and results of this paper are applicable to general space-based interferometry missions, in order to be concrete we will develop results with respect to the planned StarLight mission [7].

StarLight will be a separated-spacecraft interferometer consisting of two spacecraft - a combiner and a collector - which will be launched together into an Earth-trailing, heliocentric orbit. The mission will consist of three phases: a formation-flying experiment mode, a short-baseline monolithic-interferometer mode, and a separated-spacecraft observation mode. During this last mode, extending over a period of three months, the spacecraft will be separated by distances ranging from 40 to 600 meters, with interferometer baselines ranging from 30 to 125 meters. The combiner will combine and collect fringe data for the deconvolution of astrophysical images. The collector will act solely as a light-collecting aperture. It is currently estimated that the system will be designed to image twenty stars during this period.

In the StarLight mission, only two spacecraft are available to collect light from the stellar source and then accurately combine the light to make fringe patterns. Because of this, the spacecraft must be located so that the light path lengths differ by a known fixed amount for all configurations of the spacecraft formation. Therefore, the position of the spacecraft will be constrained such that the combiner is at the focus of a paraboloid, with the collector located on the paraboloid. Figure 3 shows the layout of the StarLight formation geometry. Notice the incoming starlight paths and where the spacecraft are located with respect to the reference paraboloid. The combiner, which has an internal delay line of two times the focal length, is positioned at the focus of a paraboloid, and the collector is located along the paraboloid to provide equal starlight path lengths. The baseline is the distance between the collector and the path of incoming starlight to the combiner. The baseline is always parallel to the stellar wavefront and perpendicular to the incoming starlight path.

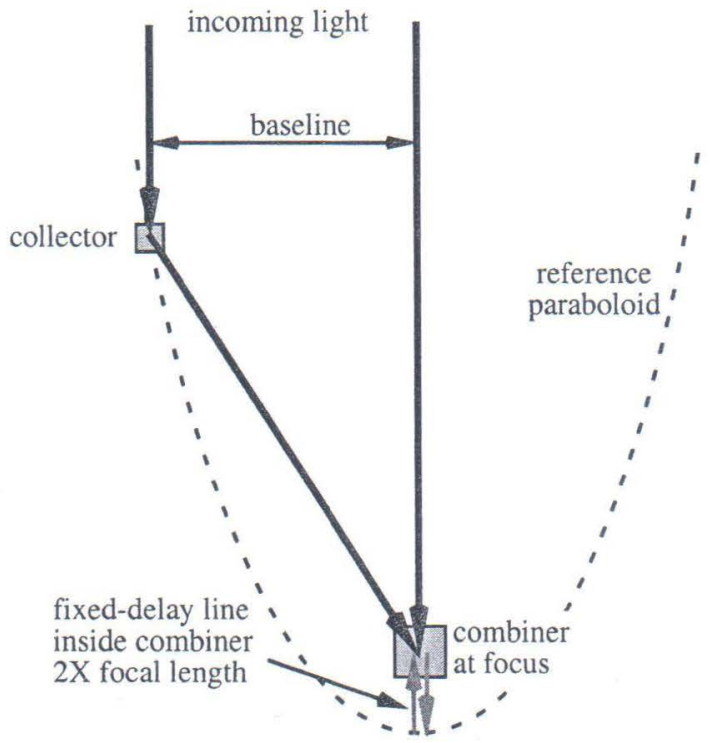

FIG. 3. Layout of the StarLight Two-Spacecraft Interferometer Concept. 
TABLE 1. StarLight System Parameters

\begin{tabular}{ll} 
Interferometry focus length & $7 \mathrm{~m}$ \\
Maximum interferometry baseline & $125 \mathrm{~m}$ \\
Minimum interferometry baseline & $30 \mathrm{~m}$ \\
Mass of combiner & $480 \mathrm{~kg}$ \\
Mass of collector & $280 \mathrm{~kg}$ \\
Maximum thrust of combiner & $7.5 \mathrm{mN}$ \\
Maximum thrust of collector & $7.5 \mathrm{mN}$ \\
Specific impulse & $60 \mathrm{sec}$ \\
\hline
\end{tabular}

The system parameters in Table 1 have been defined for the StarLight separatedspacecraft interferometry mission. Notice that the two spacecraft have equal thrust capabilities, but the combiner has more mass.

\section{Technical Approach}

A formation configuration corresponds to a particular relative position and orientation between the combiner and the collector and to a direction of the bore sight of the interferometer in the celestial sphere. For each star, there is a formation configuration for each $(u, v)$ point that needs to be imaged. A finite amount of fuel is required to transition between any two configurations. In this paper, we have assumed that the spacecraft are allowed to break formation during maneuvers. To compute the fuel cost between two configurations, we have used the results reported in [4]. Alternatively, we could have used the results reported in [3] to compute the fuel costs for the case when the spacecraft are not allowed to break formation during the maneuver.

Because the StarLight formation consists of only two spacecraft, each of the required retarget, reorient, and resize maneuvers can be carried out so that fuel consumption for the combiner and the collector are the same. However, in the general case of an $\mathrm{N}$-spacecraft formation, this is not true. For the work presented here, all maneuvers were fuel-equalizing. Fuel costs between every possible pair of configurations were computed and tabulated in a cost matrix. Overviews of the spacecraft dynamic models used and the fuel equalization problem are given below.

\section{Spacecraft and Fuel Dynamics}

The following assumptions regarding the dynamics of the spacecraft and their formation were made:

A1. The formation is in free space, but it is not in any orbit.

A2. The spacecraft are in formation before and after repositioning maneuvers, but they may break formation during repositioning.

A3. The thrusters on a single spacecraft produce thrust of finite magnitude, but they are assumed capable of providing thrust in any direction.

A4. Each spacecraft is a rigid body with time-invariant mass modeled as a point mass.

A5. The position of each spacecraft can be determined with respect to an inertial reference frame.

A6. Attitude control is not considered. For the StarLight mission, the primary actuation for attitude control is reaction wheels [7]. Fuel consumption associated with attitude maneuvers is relatively small. 
A7. The interferometric optics on the spacecraft are assumed to be at the center of the celestial sphere. Reference direction to a celestial target is not affected by the translational movements of the formation.

These assumptions are helpful in defining the problem, and they serve to simplify computations; however, they do not restrict the usefulness of the solution for application.

If the maximum thrust of a spacecraft having mass $M$ and position $\mathbf{r}$ is $\tau$, the translational and fuel dynamics for each spacecraft are given by the following equations

$$
\begin{aligned}
M \dot{\mathbf{r}} & = \begin{cases}\tau \mathbf{u} ; & f(t)>0 \\
\mathbf{0} ; & \text { otherwise }\end{cases} \\
\dot{f} & = \begin{cases}-\gamma \tau ; & f(t)>0 \text { and } \mathbf{u} \neq 0 \\
0 ; & \text { otherwise }\end{cases}
\end{aligned}
$$

where $\mathbf{u}$ is a three-dimensional unit vector given in the spacecraft body frame, and $\gamma$ is a proportionality constant calculated from the specific impulse $\left(I_{s p}\right)$ of the thruster and acceleration due to gravity $(g)$ at sea level as

$$
\gamma=\frac{1}{I_{s p} g}
$$

\section{Fuel Equalization of Maneuvers}

Under the assumption that the spacecraft are allowed to break formation during a maneuver, spacecraft motions between configurations follow straight-line paths. For a formation of two spacecraft, each of the resize, retarget, and reorient maneuvers can be performed so that fuel consumption is equal between spacecraft. For a spacecraft modeled with double-integrator translational dynamics, it is well-known that the time-optimal/fuel-optimal thrust follows a bang-off-bang profile. If a maneuver requires a spacecraft to travel the distance $\hat{y}$ in time $t_{f}$, then the pulse width for the bang-off-bang thrust profile can be calculated from [5] as

$$
t_{w}=\frac{t_{f}}{2}-\sqrt{\frac{t_{f}^{2}}{4}-\frac{M}{\tau} \hat{y}}
$$

This expression is valid provided that the transition time, $t_{f}$, is chosen so that the term under the radical remains positive.

The fuel expended during a translation maneuver is

$$
\begin{aligned}
\Delta f & =2 \gamma \tau t_{w} \\
& =2 \gamma \tau\left[\frac{t_{f}}{2}-\sqrt{\frac{t_{f}^{2}}{4}-\frac{M}{\tau} \hat{y}}\right]
\end{aligned}
$$

For each type of reposition maneuver (retarget, reorient, and resize), $\hat{y}$ for both the collector and combiner must be determined so that fuel usage is equalized while satisfying the objectives of the maneuver. For rotation maneuvers (retarget and reorient) involving two spacecraft, this is straightforward. The direction of the axis of rotation is specified by the maneuver description. The location of the rotation axis is determined to give equal fuel usage. In the case of the Starlight mission, the location 
of the rotation axis would lie along the line connecting the collector and the combiner. The rotation axis would generally be closer to the combiner because of its greater mass.

Resizing maneuvers to change the interferometer baseline can also be carried out in a fuel-equalizing manner. It is clear from Fig. 3 that the baseline can be changed by moving the collector along the paraboloid so that the position of the collector relative to the combiner is changed. Obviously, both spacecraft must move for fuel consumption to be equal. The desired change in relative position can be accomplished by moving both the collector and combiner so that their fuel usage is equal. Typically, the collector moves over greater distances than does the combiner because of its smaller mass.

The dynamic and fuel equalization equations presented above are used to calculate the fuel costs associated with repositioning the spacecraft formation. Simulations of spacecraft motion and fuel usage were carried out in Matlab. Further details of the simulations performed can be found in $[4,5]$.

\section{Formation Maneuvers - A Traveling Salesman Problem}

The amount of fuel required to move between the different configurations composing a mission can be calculated using the methods described above. From these fuel-usage computations, a cost matrix describing the cost associated with moving between any two configurations can be computed. Once the cost matrix has been determined, it is required to find a sequence, or tour, between the different configurations that minimizes the fuel cost associated with forming all of the configurations. This problem is a traveling salesman problem (TSP), which is well-known to be NP-hard ${ }^{4}$ in computational complexity. Because exact solutions to the TSP for problems of significant size are impossible to obtain, approximation methods are used. A tremendous amount of literature is devoted to approximating solutions to the TSP. Lawler, et al. [14] provide a good introduction to the TSP and some of the methods applied to its solution. Reinelt [15] offers excellent insight into choosing heuristics for finding solutions, provides a good review of present and developing methods, and gives case-study comparisons for chosen TSP instances.

To solve the TSP problem, we have used an algorithm developed by Martin, Otto, and Felten [16] called "chained local optimization" (CLO). The CLO algorithm combines simulated annealing with local search heuristics which have been shown to produce good results for the TSP. Given a starting tour for a TSP, numerous local search algorithms exist for finding a local optimum. A local search method is a way of iteratively changing a tour until no additional local changes result in a lower-cost tour. Typically a local search method is an exchange heuristic where alterations to a tour are made by deleting $k$ edges or links of the tour and reconnecting in a way to form a new complete tour. This constitutes a $k$-change. The 2-opt local search is a 2-change method and has been chosen because of its simplicity to implement.

The CLO algorithm provides an approach for searching among local optima to find the globally optimal tour. A mechanism is needed to go from one locally optimal tour to another locally optimal tour without arriving back at the same tour. Additionally, the method must push the local-optimum cost lower and lower, without getting stuck in a valley of locally optimal costs. Martin, Otto, and Felten proposed using a smart "kick" to change tour $T_{n}$ in such a way that a local-opt search could

${ }^{4}$ With respect to the TSP, NP-hard implies that any algorithm for finding the true optimal tour will have a worst-case running time that is not bounded by any polynomial in the number of tour stops. 
not return the tour to $T_{n}$. The kick is followed by a local search to get a new tour. If this new tour is less costly than tour $T_{n}$, the tour is accepted as tour $T_{n+1}$. If the new tour cost is more costly than tour $T_{n}$, a simulated annealing-like Markov process is used to accept or reject the new local-opt tour, based on a random probability function. The likelihood of accepting a more costly local-opt tour is greater in the initial stages of the search. As more iterations are taken, the probability of accepting a higher cost local-opt tour decreases.

Figure 4 depicts the operation of the CLO algorithm schematically. In Step 1, a random seed tour is selected. In Step 2, a 2-opt local search is made to find the starting tour. Once a local optimum is found, a kick is executed in Step 3 to arrive at an intermediate tour. A 2-opt local search is executed in Step 4 to arrive at a trial tour. In Step 5, the trial tour cost is compared to the starting tour cost. Since the trial cost is lower, it is accepted. If the trial cost were higher, there is some probability that it would be accepted, based on the simulated annealing approach. From the trial tour, a kick to a new intermediate tour is taken. Step 7 depicts the continuation of the algorithm until the stopping criteria are met.

\section{Results}

Optical interferometry missions involve three primary types of maneuvers-retarget, resize, and reorientation-to fill out the $(u, v)$ plane for each stellar source. One-dimensional imaging of a single source involves only resize maneuvers, while two-dimensional imaging of a single source involves resize and reorient maneuvers. Retarget maneuvers are used to move the interferometer from one source to another.

Optimizing the observation tour order can yield significant fuel-saving benefits over an ad hoc approach. Clearly, a fuel-saving strategy for an interferometry mission considering both targeting maneuvers and imaging maneuvers could result in

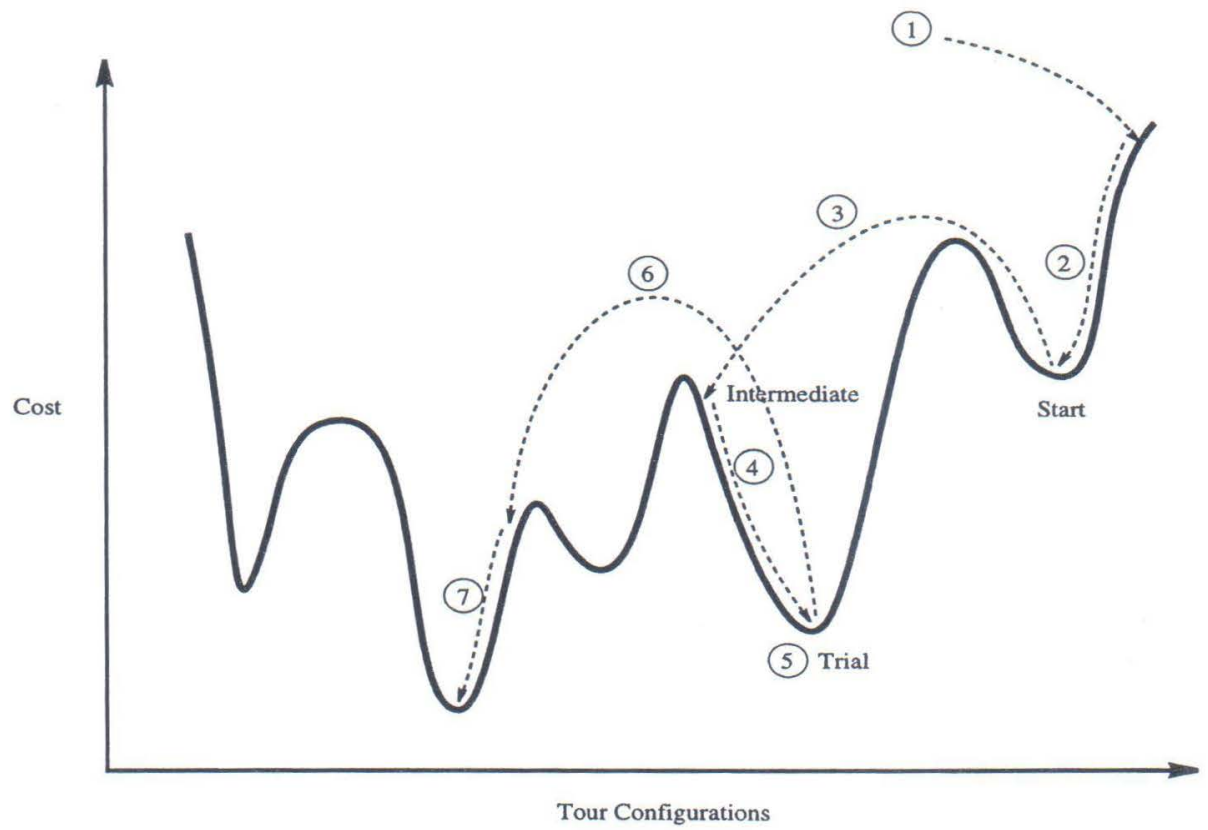

FIG. 4. Schematic Description of the Chained Local Optimization Algorithm. 
greater reductions than considering targeting maneuvers alone. Here we consider not only the targeting maneuvers for pointing the interferometer, but we also consider all of the imaging maneuvers used to fill the $(u, v)$ plane. Two types of imaging operations are considered: 1-D tours which image multiple 1-D targets, and 2-D tours, which image multiple 2-D targets. 1-D tours involve retargeting and resizing moves. 2-D tours involve retargeting, resizing, and reorienting moves.

For both 1-D and 2-D tours, the optimization results are compared to benchmark tours. Benchmark tours are generated by fully imaging each star before moving to the next star on the tour. For the 2-D tours, the image is created by resizing the interferometer baseline at each orientation before moving to the next specified orientation.

\section{1-D Imaging}

Table 2 shows five different 1-D imaging scenarios. In each case, three stars, separated by an angle $\beta$, are imaged. Three different values for $\beta$ are considered: $\pi / 16, \pi / 8$, and $\pi / 4$ radians. Baseline separation distances of ten and twenty meters are considered.

The CLO algorithm was used to determine the tour order of pointing and resizing moves that would result in minimal fuel consumption for the 1-D test cases. Results for Scenarios 1D1 and 1D2 are given in Fig. 5. A polar plot is used to depict the results, where the three radial lines represent the three observation directions. White circles along the lines represent interferometer baseline separation distance, with the baseline increasing moving away from the origin. The left-hand plot shows the fuelusage results from the benchmark tour, while the right-hand plot shows the best CLO tour with its fuel consumption for each scenario. For Scenario 1D1, the number of more costly baseline moves is minimized by the CLO algorithm. For Scenario 1D2, the result is the same as the benchmark, in which it is better to minimize the number of retarget operations. The difference between the two scenarios is the size of the retarget angle. These results suggest that when making ten meter baseline changes, targeting moves will be mixed with sampling moves when the retarget angle is $\pi / 16$ rad or less.

Results for Scenarios 1D3, 1D4, and 1D5 are given in Fig. 6. These scenarios require baseline changes of twenty meters. Again the difference between the scenarios is the size of the retarget angle. These results suggest that when making twenty meter baseline changes, targeting moves will be mixed with sampling moves when the retarget angle is $\pi / 8 \mathrm{rad}$ or less. Notice that the greatest fuel savings is realized for closely clustered observations such as the 1D3 scenario. This is expected because the pattern used in the benchmark tour is optimal for large $\beta$ angles between observation directions, as can be seen in the map of Scenario 1D5.

TABLE 2. 1-D Test Case Data

\begin{tabular}{lcc}
\hline Scenario & $\beta[\mathrm{rad}]$ & Baselines [m] \\
\hline 1D1 & $\pi / 16$ & $40,50,60,70$ \\
1D2 & $\pi / 8$ & $40,50,60,70$ \\
1D3 & $\pi / 16$ & $40,60,80,100$ \\
1D4 & $\pi / 8$ & $40,60,80,100$ \\
1D5 & $\pi / 4$ & $40,60,80,100$ \\
\hline
\end{tabular}




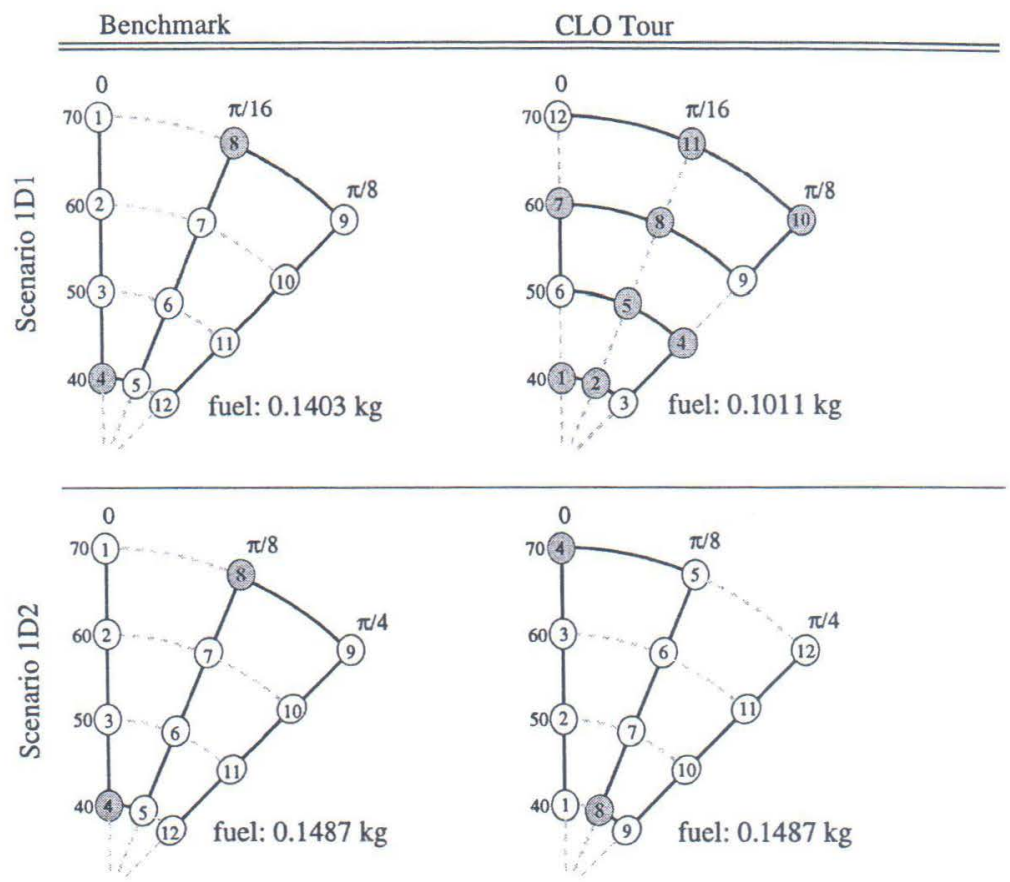

FIG. 5. Tour Map for Scenarios 1D1 and 1D2.

From these results, it is clear that when the separation angle between stars is large, it is best to point at a star and perform all the baseline resizing moves before moving on to the next star. Conversely, when the separation angle between stars is relatively small, less fuel is consumed when the baseline is held fixed and all the stars are targeted before changing the baseline.

Guidelines for how these 1-D moves can be sequenced in the best way can be developed by comparing the maximum retarget and resize costs. The maximum retarget cost, $R T_{\max }$, is defined as the cost to retarget to the next closest star when the formation is at its maximum baseline. The maximum resize cost, $R S_{\max }$, is defined as the cost to resize the formation from its maximum baseline to the next smallest baseline. Because the spacecraft are constrained to move along the reference paraboloid to resize the formation, the maximum resize cost will result when the spacecraft are at the largest baseline separation.

The maximum retarget cost and resize cost for Scenarios 1D1 to 1D5 have been calculated; they are shown with the cost inequalities in Table 3. These cost inequalities suggest the priority of maneuvers to construct the fuel-optimal tour.

Figure 7 shows an example of how a 1-D imaging Scenario might be constructed using only the maximum retarget and resize costs $\left(R T_{\max }\right.$ and $\left.R S_{\max }\right)$ as a guide. The numbers inside of the circles represent the tour order. Gray-shaded circles indicate that the next maneuver is a retarget. By comparing $R T_{\max }$ and $R S_{\max }$, a priority ranking can be given to the lower cost maneuver. When it is more costly to retarget than resize $\left(R T_{\max }>R S_{\max }\right)$, as Fig. 7a shows, a fuel-minimal tour should resize until it must retarget. In this case, a fuel-optimal tour results when the number of more costly moves (retargets) is minimized. There must be at least two retargets to complete the mission. Alternatively, when resizing is more expensive than retargeting 

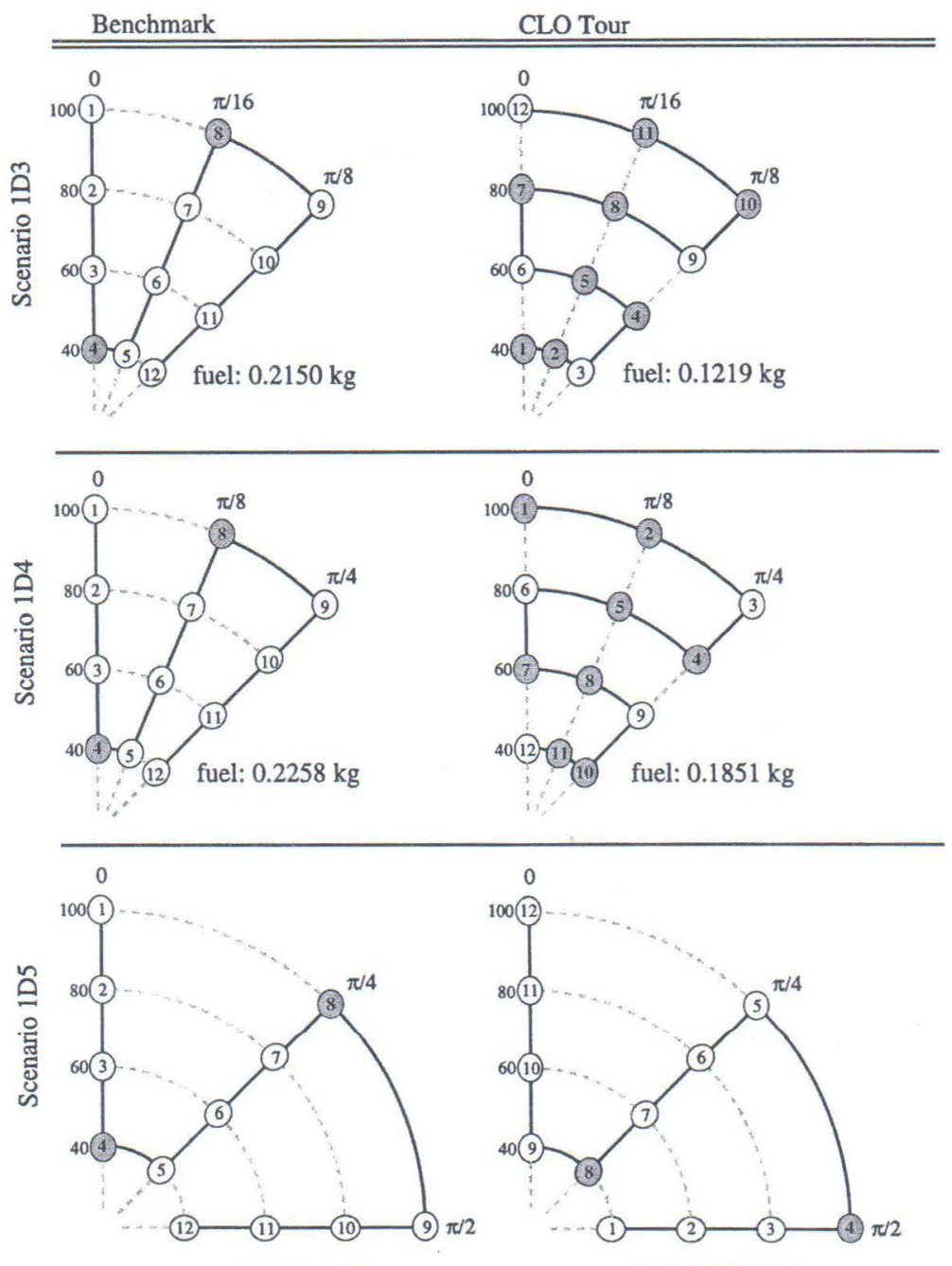

fuel: $0.2406 \mathrm{~kg}$

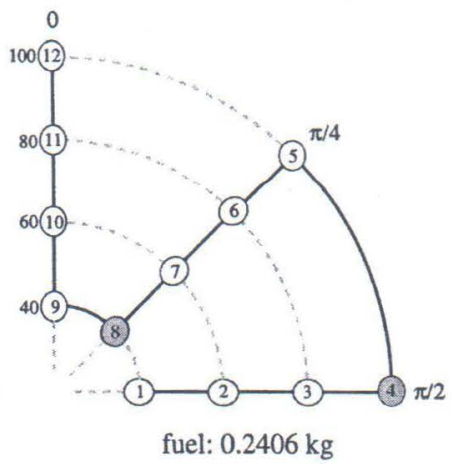

FIG. 6. Tour Map for Scenarios 1D3, 1D4, and 1D5.

$\left(R S_{\max }>R T_{\max }\right)$ as Fig. $7 \mathrm{~b}$ shows, the tour should retarget to each star in the cluster before changing baselines. A minimum of three resize maneuvers is required, as shown in Fig. $7 b$.

When the sampling pattern guidelines are applied to the five 1-D test cases, the results are identical to those obtained by the CLO algorithm, with the exception of

TABLE 3. 1-D Test Case Maximum Retarget and Resize Costs

\begin{tabular}{lccc}
\hline Scenario & $R T_{\max }[\mathrm{kg}]$ & $R S_{\max }[\mathrm{kg}]$ & Priority \\
\hline 1D1 & 0.0126 & 0.0145 & $R S_{\max }>R T_{\max }$ \\
1D2 & 0.0178 & 0.0145 & $R T_{\max }>R S_{\max }$ \\
1D3 & 0.0178 & 0.0239 & $R S_{\max }>R T_{\max }$ \\
1D4 & 0.0251 & 0.0239 & $R T_{\max }>R S_{\max }$ \\
1D5 & 0.0351 & 0.0239 & $R T_{\max }>R S_{\max }$ \\
\hline
\end{tabular}




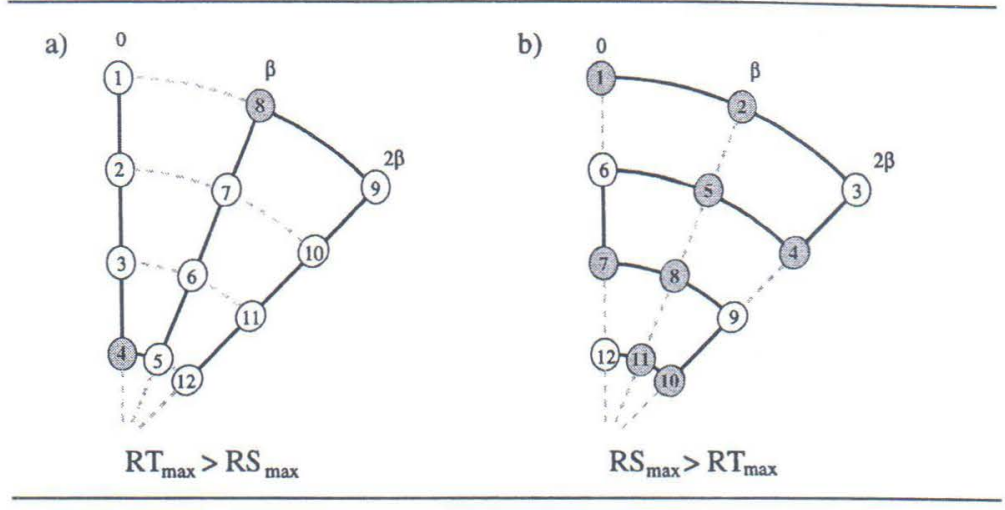

FIG. 7. Sampling Pattern Guide for 1-D Imaging.

Scenario 1D4. The guide tour from Scenario 1D4 was more costly than the tour found with CLO. From Table 3, it can be seen that for Scenario 1D4, $R T_{\max }$ is only slightly larger than $R S_{\max }$. Thus, the sampling guidelines suggest that the number of baseline resize moves should be minimized. Because the guidelines only consider the maximum retarget or resize cost and not the total cost of all of the possible retarget or resize moves, it is possible that the guidelines may not lead to the optimal fuel savings when $R T_{\max }$ and $R S_{\max }$ are close in value. It should be noted that under these conditions, the fuel usage for the CLO tour and guideline tour differ only slightly.

\section{2-D Imaging}

The 2-D test-case scenarios were designed to investigate fuel saving strategies when retargeting, reorienting, and resizing moves are involved. The test-case scenarios investigated are shown in Table 4.

The CLO algorithm was used to find tours for each of these eight scenarios. Following the run of each scenario, the mission was simulated using the CLOsuggested tour to determine the amount of fuel expended. Figures 8, 9, and 10 show CLO results for Scenarios 2D1, 2D2, and 2D6. In each figure, the resultant CLO tour and its fuel consumption is shown with a benchmark tour and its fuel consumption for comparison. These figures use a polar plot similar to those used for the 1-D tours; however, the representation is different. Here, two plots are given, where each plot represents an observation in the directions shown by the bold arrows in the upper left corner of the figure. The radial lines in each plot represent the orientation of

TABLE 4. 2-D Test Case Data

\begin{tabular}{|c|c|c|c|}
\hline Scenario & $\beta[\mathrm{rad}]$ & Baselines [m] & Orientations [rad] \\
\hline 2D1 & $\pi / 8$ & $40,60,80$ & $0, \pi / 8, \pi / 4,3 \pi / 8, \pi / 2$ \\
\hline $2 \mathrm{D} 2$ & $\pi / 4$ & $40,60,80$ & $0, \pi / 8, \pi / 4,3 \pi / 8, \pi / 2$ \\
\hline $2 \mathrm{D} 3$ & $3 \pi / 8$ & $40,60,80$ & $0, \pi / 8, \pi / 4,3 \pi / 8, \pi / 2$ \\
\hline 2D4 & $\pi / 2$ & $40,60,80$ & $0, \pi / 8, \pi / 4,3 \pi / 8, \pi / 2$ \\
\hline 2D5 & $\pi / 4$ & $40,45,50$ & $0, \pi / 4, \pi / 2,3 \pi / 4, \pi$ \\
\hline 2D6 & $\pi / 16$ & $40,60,80$ & $0, \pi / 4, \pi / 2,3 \pi / 4, \pi$ \\
\hline $2 \mathrm{D} 7$ & $\pi / 12$ & $40,45,50$ & $0, \pi / 4, \pi / 2,3 \pi / 4, \pi$ \\
\hline $2 \mathrm{D} 8$ & $\pi / 16$ & $40,50,60$ & $0, \pi / 4, \pi / 2,3 \pi / 4, \pi$ \\
\hline
\end{tabular}



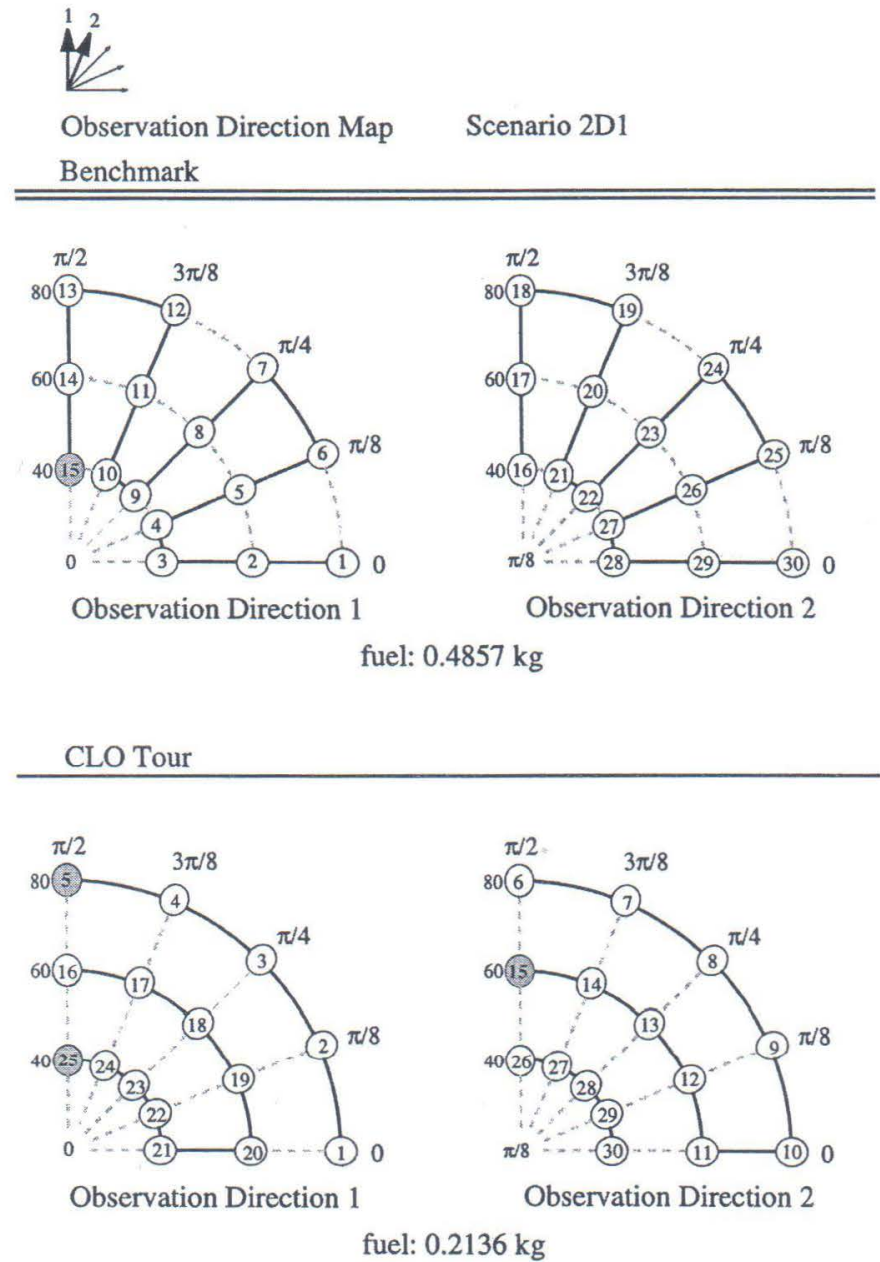

FIG. 8. Tour Map for Scenario 2 D1.

the spacecraft pair about the different observation directions. The white circles along the radial lines represent the baseline separation distance as before. For the benchmark tour, each star is fully imaged before moving to the next star, with baseline resize moves being carried out at each orientation before moving to the next orientation. The mission durations for the CLO tours are adjusted to take the same amount of time as the benchmark tour to execute the maneuvers required to perform the mission objectives.

Scenarios 2D1, shown in Fig. 8, images two stars separated by a $\beta$ angle of $\pi / 8 \mathrm{rad}$. The CLO tour samples at all orientations with a single baseline for both stars before moving to the next baseline. Note the fuel savings realized by the CLO tour. The figure demonstrates that for stars closely clustered together, mixing targeting and sample maneuvers provides a large reduction in fuel consumption as compared to that of the benchmark tour.

The 2D2 scenario shown in Fig. 9 images two stars separated by a larger $\beta$ angle of $\pi / 4 \mathrm{rad}$. Like the baseline tour, the CLO tour also entirely samples one star, but 


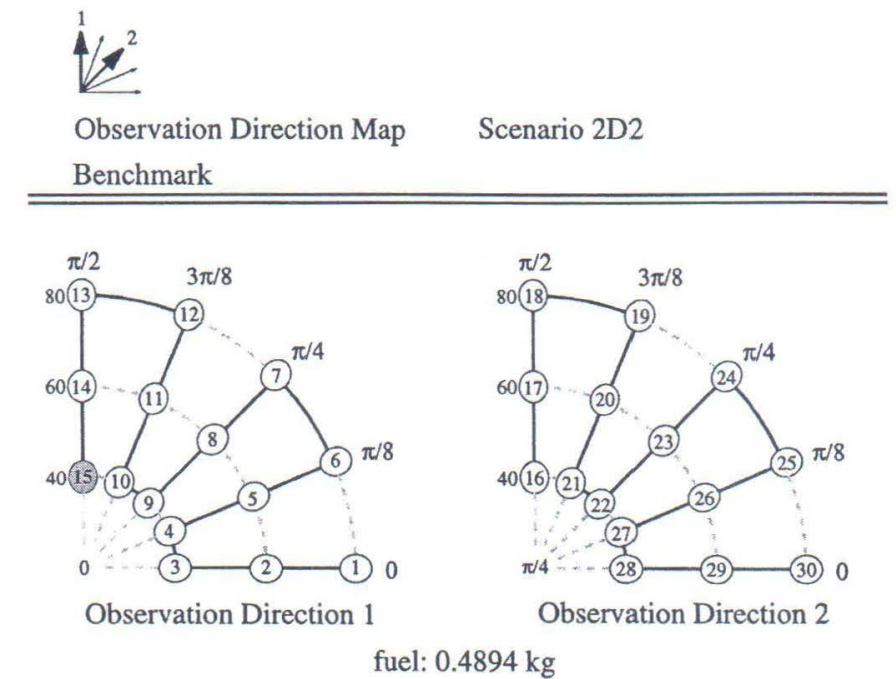

fuel: $0.4894 \mathrm{~kg}$

\section{CLO Tour}

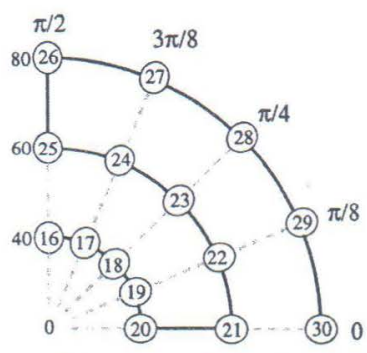

Observation Direction 1

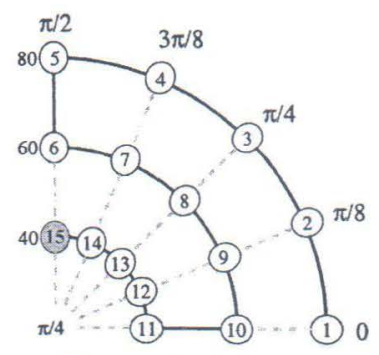

Observation Direction 2

fuel: $0.2242 \mathrm{~kg}$

FIG. 9. Tour Map for Scenario 2D2.

it samples at all orientations of that star with a single baseline before changing to the next baseline. Even though each star is imaged separately, the CLO tour is still more fuel-efficient.

In Fig. 10, CLO results are shown for Scenario 2D6, where $\beta=\pi / 16 \mathrm{rad}$ is small relative to the angle separating the orientation directions $(\pi / 4 \mathrm{rad})$. The CLO tour switches observation directions as each image sample is taken. Because baseline changes are the most costly maneuvers, only two baseline changes are made in imaging the two stars. As with Scenario 2D1, significant fuel savings are achieved by mixing targeting and sampling maneuvers.

The maximum retarget, reorient, and resize cost for Scenarios 2D1 through 2D8 have been calculated; they are shown in Table 5 with the priority cost inequalities. These cost inequalities suggest the priority of maneuvers to construct the fuel optimal tour. $R T_{\max }$ and $R S_{\max }$ are calculated the same way as for 1-D imaging. The maximum reorient cost, $R O_{\max }$, is defined as the cost to reorient to the next closest orientation when the formation is at its maximum baseline. This will be the 


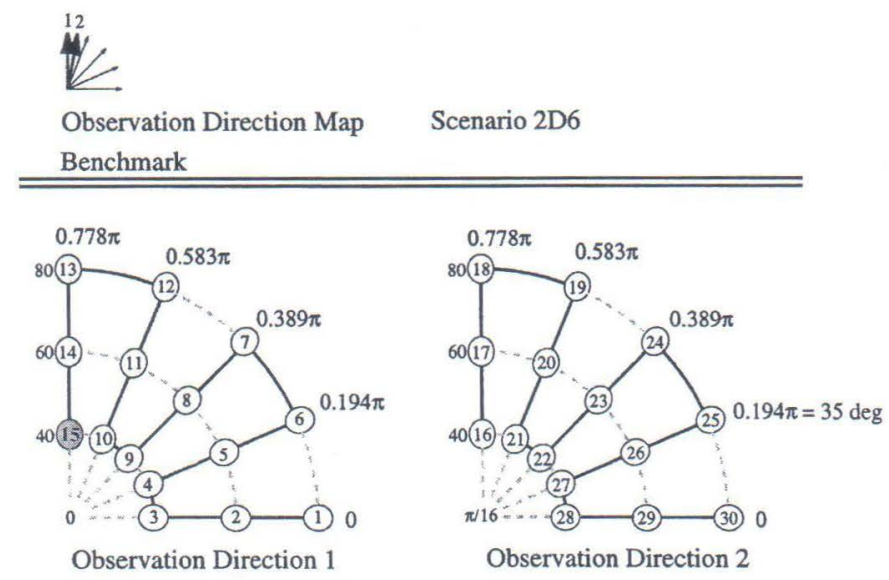

fuel: $0.5129 \mathrm{~kg}$

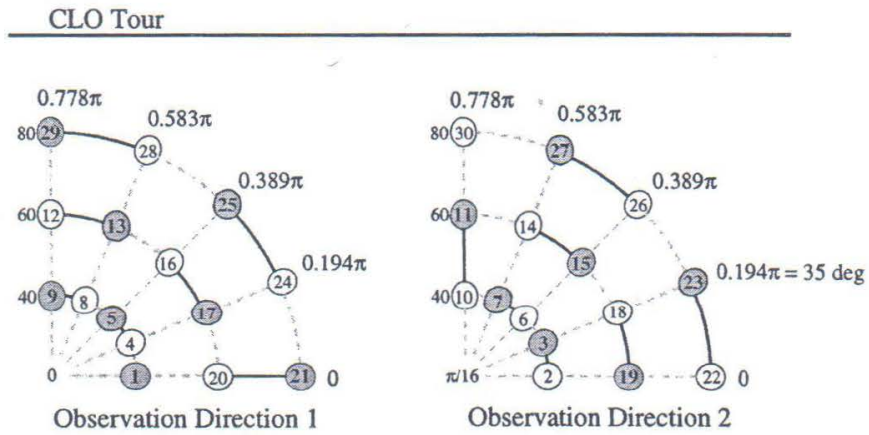

fuel: $0.2564 \mathrm{~kg}$

FIG. 10. Tour Map for Scenario 2D6.

maximum reorient cost, since the spacecraft must travel farther to reorient at larger baselines.

By examining the priority cost inequalities for Scenarios 2D2, 2D3, and 2D4, it is expected that the optimal sampling patterns for Scenarios 2D3 and 2D4 would be the same as the optimal pattern for Scenario 2D2. This has been confirmed by CLO results for these scenarios.

By comparing the priority cost inequalities with the CLO results obtained for each of the 2-D scenarios, guideline tours can be constructed. For example, in Scenario 2D1, reorient moves use the least amount of fuel. Accordingly, the tour remains on the same target and baseline until no further reorient moves can be made (tour Stop 5 of Fig. 8). At this point, the priority suggests that it is cheaper to retarget than to resize, so a retarget move is made from Stop 5 to Stop 6. The tour then reorients from Stop 6 through Stop 10, where the first baseline change is made. Since $R S_{\max }$ has the lowest priority for this scenario, the objective is to make the minimum number of resize moves while completing the mission requirements.

Using such reasoning, guideline tours can be developed for every possible cost priority. The guideline tours in Fig. 11 result in the same sampling patterns as obtained from CLO algorithm for Scenarios 2D1 through 2D8. The guideline tours ad- 
TABLE 5. 2-D Test Case Maximum Retarget, Reorient, and Resize Costs

\begin{tabular}{lcccc}
\hline Scenario & $R T_{\max }[\mathrm{kg}]$ & $R O_{\max }[\mathrm{kg}]$ & $R S_{\max }[\mathrm{kg}]$ & Priority \\
\hline 2D1 & 0.0202 & 0.0117 & 0.0212 & $R S_{\max }>R T_{\max }>R O_{\max }$ \\
2D2 & 0.0283 & 0.0117 & 0.0212 & $R T_{\max }>R S_{\max }>R O_{\max }$ \\
2D3 & 0.0341 & 0.0117 & 0.0212 & $R T_{\max }>R S_{\max }>R O_{\max }$ \\
2D4 & 0.0385 & 0.0117 & 0.0212 & $R T_{\max }>R S_{\max }>R O_{\max }$ \\
2D5 & 0.0182 & 0.0129 & 0.0088 & $R T_{\max }>R O_{\max }>R S_{\max }$ \\
2D6 & 0.0143 & 0.0164 & 0.0212 & $R S_{\max }>R O_{\max }>R T_{\max }$ \\
2D7 & 0.0107 & 0.0129 & 0.0088 & $R O_{\max }>R T_{\max }>R S_{\max }$ \\
2D8 & 0.0109 & 0.0142 & 0.0134 & $R O_{\max }>R S_{\max }>R T_{\max }$ \\
\hline
\end{tabular}

dress all the possible scenarios for 2-D imaging of multiple stars and provide systematic methods for sweeping out the sampling pattern in a fuel-efficient way.

Figures 11a through 11f show how a 2-D observation sequence might be constructed by comparing the maximum retarget, reorient, and resize costs. Scenario 2D1, discussed previously, corresponds to Fig. 11c. Guide tours for each of the other combinations of maximum repositioning costs, $R T_{\max }, R O_{\max }$, and $R S_{\max }$ are

a)
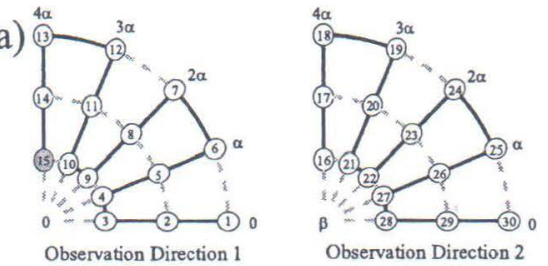

$\mathrm{RT}_{\max }>\mathrm{RO}_{\max }>\mathrm{RS}_{\max }$ b)
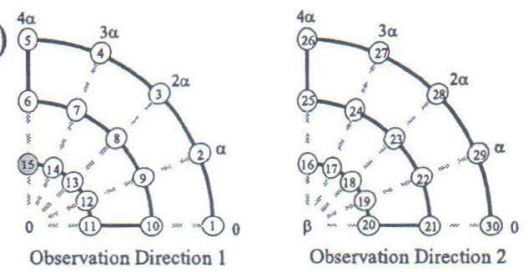

$\mathrm{RT}_{\max }>\mathrm{RS}_{\max }>\mathrm{RO}_{\max }$ c)

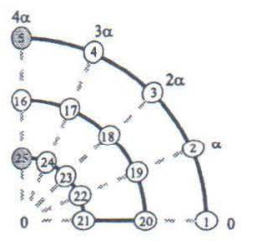

Observation Direction 1

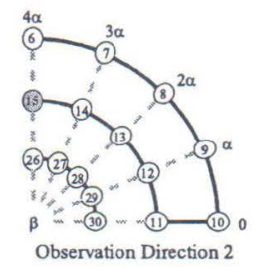

$\mathrm{RO}_{\max }$ d)

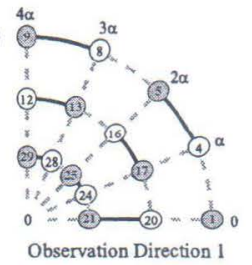

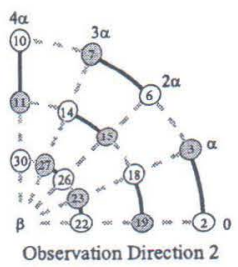

Observation Direction 2

$\mathrm{RS}_{\max }>\mathrm{RO}_{\max }>\mathrm{RT}_{\max }$

e)
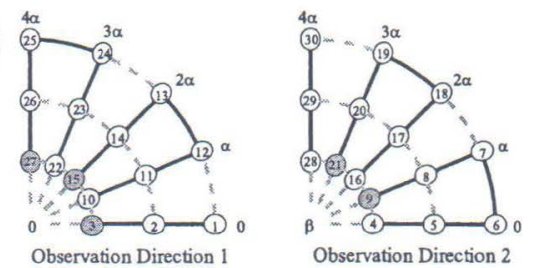

$\mathrm{RO}_{\max }>\mathrm{RT}_{\max }>\mathrm{RS}_{\max }$ f)
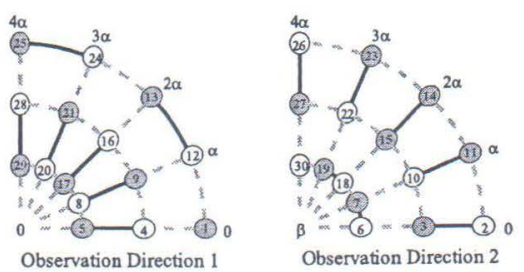

$$
\mathrm{RO}_{\max }>\mathrm{RS}_{\max }>\mathrm{RT}_{\max }
$$

FIG. 11. Sampling Pattern Guide for 2-D Imaging. 
determined following the same priority path system. For example, in Fig. $11 \mathrm{f}$ because $R O_{\max }>R S_{\max }>R T_{\max }$, the number of reorient moves should be minimized followed by the number of resize moves. The minimum number of reorient moves is four, and the minimum number of resize moves is ten. Clearly, Fig. 11 can help determine how the sampling pattern should be mapped out to fill the $(u, v)$ plane in the most fuel-efficient way. The guidelines can be used to form a final sampling plan for a mission or as an initial condition for further optimization studies. Table 6 summarizes the minimum number of basic maneuvers that must be performed for each of the six priority paths to construct fuel-minimal tours. Since there are thirty tour stops, and only basic maneuvers can be used to travel between stops, twenty-nine maneuvers must be performed. Therefore, the sum of all retarget, reorient, and resize maneuvers will be twenty-nine. Notice in this table that the number of basic maneuvers for each pattern matches the priority inequality.

When sampling with the same baseline-change and $\beta$ values, the results of the 2-D scenarios are consistent with the results from the 1-D scenarios. Notice that the CLO algorithm chose to mix targeting and sampling maneuvers when the retargeting $\beta$ angle was $\pi / 8 \mathrm{rad}$, as in Scenarios $1 \mathrm{D} 4$ and $2 \mathrm{D} 1$. For $\beta$ angles of $\pi / 4 \mathrm{rad}$, the tour images each star separately as shown by Scenarios 1D5 and 2D2.

\section{Fuel Savings}

Table 7 shows the percentage of savings of the guide tour below the benchmark tour for each of the scenarios tested. Where the percent savings is 0.0 , the benchmark tour is the same as the guide tour. Mixing of targeting and sampling moves took place on Scenarios 2D1, 2D6, 2D7, and 2D8. Notice that even when no mixing occurs (e.g., Scenarios 2D2, 2D3, and 2D4), significant savings below the benchmark tour are still realized. This is accomplished by optimizing the order of reorient and resize moves. This suggests that even if the stars are not tightly clustered and only one star is imaged at a time, the sampling pattern is still an important consideration for fuel efficiency.

\section{Application to the StarLight Mission}

The sampling guidelines presented in this paper can be used to establish the sequence of retarget, reorient, and resize moves for the StarLight mission. Once the celestial targets and $(u-v)$ plane imaging points have been defined for the mission, the maneuver costs $R T_{\max }, R O_{\max }$, and $R S_{\max }$ can be calculated. Based on the priorities of the maneuvers, the appropriate imaging pattern can be chosen for a pair or group of targets using the guidelines of Figs. 7 and 11. For closely clustered targets, mixing of targeting and sampling moves may be required to minimize fuel con-

TABLE 6. Number of Basic Maneuvers for Fuel-Minimal Tours

\begin{tabular}{llrrr}
\hline Pattern & Priority & Retarget & Reorient & Resize \\
\hline$a$ & $R T_{\max }>R O_{\max }>R S_{\max }$ & 1 & 8 & 20 \\
$b$ & $R T_{\max }>R S_{\max }>R O_{\max }$ & 1 & 24 & 4 \\
$c$ & $R S_{\max }>R T_{\max }>R O_{\max }$ & 3 & 24 & 2 \\
$d$ & $R S_{\max }>R O_{\max }>R T_{\max }$ & 15 & 12 & 2 \\
$e$ & $R O_{\max }>R T_{\max }>R S_{\max }$ & 5 & 4 & 20 \\
$f$ & $R O_{\max }>R S_{\max }>R T_{\max }$ & 15 & 4 & 10 \\
\hline
\end{tabular}


TABLE 7. Percent Savings of Test Cases Below Benchmark

\begin{tabular}{lc}
\hline Scenario & Percent Savings \\
\hline 1D1 & 38.8 \\
1D2 & 0.0 \\
1D3 & 76.4 \\
1D4 & 0.0 \\
1D5 & 0.0 \\
2D1 & 127.4 \\
2D2 & 118.3 \\
2D3 & 117.2 \\
DD & 116.6 \\
D5 & 0.0 \\
2D6 & 100.0 \\
2D7 & 11.8 \\
2D8 & 39.9 \\
\hline
\end{tabular}

sumption. For targets that are imaged one at a time, the guidelines provide sequences for resize and reorient maneuvers that can result in significant fuel savings. The sampling sequences developed from the guidelines can be used directly for the mission, or they can be used to provide a good initial sequence for more advanced tour-optimization studies.

\section{Conclusions}

Fuel-optimal tour strategies have been developed for dual spacecraft interferometry missions using chained local optimization. These tour strategies show that significant fuel savings can be realized by combining retargeting moves, used to point the spacecraft formation toward the stellar source, with imaging moves, used to position the formation about the observation direction. Comparing fuel-optimal tours for imaging two stars with a benchmark tour showed fuel savings of up to 127 percent. The fuel savings were most significant for test cases involving closely clustered stars. For stars with large separation angles, targeting moves are carried out independent of the imaging moves. Even so, significant fuel savings can be achieved by considering the sequence of the imaging maneuvers alone. From the optimization results, sampling pattern guidelines based on easily obtained targeting and imaging metrics have been developed. These guidelines provide a convenient and direct means for planning fuel-efficient, dual-spacecraft interferometry mission tours.

\section{Acknowledgments}

The third author was partially funded by the Jet Propulsion Laboratory, California Institute of Technology, under Grant 96-1254.

\section{References}

[1] SHAO, M. and COLAVITA, M. M. "Long-Baseline Optical and Infrared Stellar Interferometry," Annual Review of Astronomy and Astrophysics, Vol. 30, pp. 457-498, 1992.

[2] JOSHI, SANJAY S. "An Informal Introduction to Synthetic Aperture Imaging," Interoffice Memorandum 3450-98-0004, Jet Propulsion Laboratory, Pasadena, CA 91109, February 1998.

[3] BEARD, RANDAL W., MCLAIN, TIMOTHY W., and HADAEGH, FRED Y. "Fuel Optimization for Constrained Rotation of Spacecraft Formations," AIAA Journal of Guidance, Control, and Dynamics, Vol. 23, No. 1, pp. 1-8, January-February 2000. 
[4] BEARD, RANDAL W. and HADAEGH, FRED Y. "Fuel Optimized Rotation for Satellite Formations in Free Space," Presented at the American Control Conference, San Diego, CA, 1999.

[5] BAILEY, CHRISTOPHER A. Fuel Saving Schemes for Separated Spacecraft Flying in Formation, Master's thesis, Brigham Young University, Department of Mechanical Engineering, December 1999.

[6] BLACKWOOD, G. H., DUBOVITSKY, S., LINFIELD, R. P., and GORHAM, P.W. "Interferometer Instrument Design for New Millennium Deep Space 3," Presented at the SPIE International Symposium on Astronomical Telescopes and Instruments, Kona, HI, March 1998.

[7] DEININGER, W. D., WEISS, M. A., WEIMER, D. J., HOFFMAN, C. N., CLEVEN, G. C., PATEL, K. C., LINFIELD, R. P., and LIVESAY, L. L. "Description of the StarLight Mission and Spacecraft Concepts," Proceedings of the IEEE Aerospace Conference, 2001.

[8] WANG, P. K. C. and HADAEGH, F.Y. "Coordination and Control of Multiple Microspacecraft Moving in Formation," The Journal of the Astronautical Sciences, Vol. 44, No. 3, pp. 315-355, 1996.

[9] WANG, P. K. C., HADAEGH, F. Y., and LAU, K. "Synchronized Formation Rotation and Attitude Control of Multiple Free-Flying Spacecraft," AIAA Journal of Guidance, Control and Dynamics, Vol. 22, No. 1, pp. 28-35, January 1999.

[10] MCINNES, COLIN R. "Autonomous Ring Formation for a Planar Constellation of Satellites," Journal of Guidance, Control and Dynamics, Vol. 18, No. 5, pp. 1215-1217, 1995.

[11] LAWTON, JONATHAN, BEARD, RANDAL W., and HADAEGH, FRED Y. "An Adaptive Control Approach to Satellite Formation Flying with Relative Distance Constraints," Proceedings of the American Control Conference, pp. 1545-1549, San Diego, CA, June 1999.

[12] BEARD, RANDAL W., LAWTON, JONATHAN, and HADAEGH, FRED Y. "A Coordination Architecture for Spacecraft Formation Control," IEEE Control Systems Technology, Vol. 9, No. 6, pp. 777-790, 2001.

[13] KAPILA, VIKRAM, SPARKS, ANDREW G., BUFFINGTON, JAMES M., and YAN, QIGUO "Spacecraft Formation Flying: Dynamics and Control," Proceedings of the American Control Conference, pp. 4137-4141, June 1999.

[14] LAWLER, E. L., LENSTRA, J. K., KAN, A. H. G. RINNOOY, and SHMOYS, D. B. editors The Traveling Salesman Problem, John Wiley \& Sons, 1985.

[15] REINELT, GERHARD The Traveling Salesman: Computational Solutions for TSP Applications, Springer-Verlag, 1994.

[16] MARTIN, OLIVIER C., OTTO, STEVE W., and FELTEN, EDWARD W. "Large-Step Markov Chains for the Traveling Salesman Problem," Complex Systems, Vol. 5, No. 3, p. 299, 1991. 